\title{
Evolution law for shock strength in simple elastic structures
}

\author{
Slawomir Kosiński \\ Dept. of Mechanics of Materials $K-61$, \\ Lódź University of Technology, Al. Politechniki 6, 93-590 Lódź, Poland. \\ Phone/Fax: (048) (042) 313551. \\ E-mail: slawek@kmm-sun.p.lod.edu.pl
}

\begin{abstract}
The longitudinal shock wave propagates in the semi-infinite thin elastic layer and the material region in front of it is unstrained and at rest. Rods and layers are simple elastic structures and they are usually modeled as one-dimensional objects. In the paper a one-dimensional continuum theory with one internal scalar variable is used to describe the thin layer with propagating shock. In such a way the effect of small but finite transversal dimensions can be considered. From the singular surface theory and the equations of motion results a set of coupled evolution equations for the shock amplitude and the amplitudes of higher order discontinuities which accompany the shock. Using the perturbation method with a small perturbation parameter - the initial amplitude of the shock, we obtain the approximate solution of the problem; the longitudinal (or quasilongitudinal) shock propagates accompanied by the discontinuity of third order connected with the transverse motion.
\end{abstract}

\section{Keywords}

shock waves, singular surface theory, nonlinear elastic material, evolution laws, perturbation methods, unidimensional theory

\section{INTRODUCTION}

In the simple nonlinear theories for wave propagation a slender layer can be treated as onedimensional object endowed with structure i.e. additionally one internal scalar variable is introduced. This variable represent the axisymmetric motion and includes the effects connected with the transverse dimension (Wright, 1981). In this paper we wish to apply this approximation method to the studies of shock wave propagation in slender elastic layer. For above problem we are interested in the differences in quality between the averaged unidimensional theory for 3-D slender elastic layer (Wright, 1981) and the simplest 1-D theory for nonlinear wave propagation in thin uniform layer (Fu and Scott, 1989). The simplest perturbation solution of the problem gives the uncoupled with the transverse motion system of 
equations for functions connected with the longitudinal motion only, identical as in $\mathrm{Fu}$ and Scott (1989). The influence of transversal shear and inertia gives additional effects for shock propagation. According to the boundary conditions, the longitudinal shock wave corresponding with the main motion, propagates accompanied by either third order discontinuity (see Figure 1) or third order discontinuity with transversal shock (see Figure 2). We shall work with the isentropic approximation and with nonlinear Murnaghan material. Numerical analysis (Kosiński, 1995) shows that only shocks of a relatively small (of order up to $10^{-3}$ ) strength can propagate in Murnaghan material. Thus it is reasonable then to consider perturbation methods as a means of finding an approximate solution of the evolution problem.

\section{BASIC EQUATIONS}

Suppose that a plane shock wave propagates through a thin elastic layer in the direction of the $X_{1}$ axis in region $X_{1}>0$. See Figure 1. The axial symmetric motion (according to $X_{2}$ axis) of the semi-infinite layer is assumed in the form :

$$
\begin{gathered}
x_{1}=X_{1}+u_{1}\left(X_{1}, t\right), \quad x_{2}=X_{2}+f\left(X_{2}\right) u_{2}\left(X_{1}, t\right), \\
x_{3}=X_{3},
\end{gathered}
$$

where: $f\left(X_{2}\right)$ is an arbitrary odd function $f \in C^{1}(\langle-h, h\rangle \rightarrow \mathrm{R})$, and functions $u_{k} \in C^{3}(\langle 0, \infty) \times\langle 0, \infty) \rightarrow R) k=1,2$ (see (16)) $x_{i}(i=1,2,3)$ is the position at time $t$ of a particle which is at the position $X_{\alpha}(\alpha=1,2,3)$ in the undeformed configuration $\mathrm{B}_{\mathrm{R}}$ and the principal assumption (Wright, 1981)) is made that this motion occurs without application of perceptible contact forces to the lateral boundaries $X_{2}= \pm$ h (see Figure 1). Here and throughout this paper we assume the simplest form of $f\left(X_{2}\right)=X_{2}$. The displacements $U_{1}, U_{2}$ the strains $e_{1}$, $e_{2}$ and the particle velocities $v_{1}, v_{2}$ are given by

$$
\begin{aligned}
& U_{1}=x_{1}-X_{1}=u_{1}\left(X_{1}, t\right), \quad U_{2}=x_{2}-X_{2}=X_{2} u_{2}\left(X_{1}, t\right) \\
& \dot{x}_{1}=v_{1}=\dot{u}_{1}\left(X_{1}, t\right), \dot{x}_{2}=v_{2}=X_{2} \dot{u}_{2}\left(X_{1}, t\right) \quad e_{1}=u_{1,1}, e_{2}=u_{2,1}
\end{aligned}
$$

For the assumed motion (1) the deformation gradient is given by

$$
\left[x_{i \alpha}\right]=\left[\begin{array}{ccc}
1+u_{1,1}\left(X_{1}, t\right) & 0 & 0 \\
X_{2} u_{2,1}\left(X_{1}, t\right) & 1+u_{2}\left(X_{1}, t\right) & 0 \\
0 & 0 & 1
\end{array}\right],
$$

The material region $\mathrm{F}$ in front of the propagating shock $\Sigma\left(X_{1}, t\right)$ is initially unstrained and at rest, the region $B$ behind of it (comp. (1)) is in a state of plane strain deformation. The jumps of both displacements $U_{1}$ and $U_{2}$ on the discontinuity surface $\Sigma\left(X_{1}, t\right)$ are equal zero, for this reason because of continuity we obtain that the jumps of the functions $u_{1}\left(X_{1}, t\right), u_{2}\left(X_{1}, t\right)$ are also equal zero

$$
\left[U_{1}\right]=0 \Rightarrow\left[u_{1}\right]=0, \quad\left[U_{2}\right]=0 \Rightarrow\left[u_{2}\right]=0,
$$


but we assume that the both derivatives $u_{1,1}, u_{2,1}$ can suffer a jump on the discontinuity surface $\Sigma\left(X_{1}, t\right)$. The deformation gradient on both sides of $\Sigma\left(X_{1}, t\right)$ has the form

$\left[x_{i \alpha}\right]^{B}=\left[\begin{array}{ccc}1+u_{1,1}\left(X_{1}, t\right) & 0 & 0 \\ X_{2} u_{2,1}\left(X_{1}, t\right) & 1 & 0 \\ 0 & 0 & 1\end{array}\right], \quad\left[x_{i \alpha}\right]^{F}=\delta_{i \alpha}$

\section{AVERAGED EQUATION OF MOTION}

The equations of motion for the deformation gradient (3) are reduced to the system of equations for plane deformation:

$T_{R 11,1}+T_{R 12,2}=\rho_{R} \ddot{u}_{1}$

$T_{R 21,1}+T_{R 22,2}=\rho_{R} X_{2} \ddot{u}_{2}$

$T_{R 33,3}=0$, (identity)

where the stress component $T_{R i \alpha}$ used here are the unsymmetric Piola-Kirchhoff stress components and $\rho_{R}$ is the density in $B_{R}$. The boundary conditions on the lateral surfaces of the layer require that two stress components vanish.

$\left.\begin{array}{l}t_{i}=T_{R i \alpha} K_{\alpha}=0, \\ K_{\alpha}=(0,1,0)\end{array}\right\} \Rightarrow T_{R 12}=T_{R 22}=0$ for $X_{2}= \pm h$

The equation (6) ${ }_{2}$ is multiplied by $X_{2}$ and we average now the both equations of motion (6) over the unit length cross section $A\left(A=1 \times 2 h\right.$ has unit length in the direction of the $X_{3}$ axis and is perpendicular to the $X_{1}$ axis). After integration of (6) and for the stress free lateral surfaces $\left.T_{R 22}\right|_{-h} ^{h}=\left.T_{R 12}\right|_{-h} ^{h}=0$ we obtain equations

$$
\begin{aligned}
& \frac{\partial}{\partial X_{1}}\left(\frac{1}{2 h} \int_{-h}^{h} T_{R 11} d X_{2}\right)=\rho_{R} \ddot{u}_{1}, \\
& \frac{\partial}{\partial X_{1}}\left(\begin{array}{c}
\frac{1}{2 h} \int_{-h}^{h} X_{2} T_{R 21} d X_{2} \\
-\frac{1}{2 h} \int_{-h}^{h} T_{R 22}=\rho_{R} \ddot{u}_{2} \frac{h^{2}}{3}
\end{array}\right.
\end{aligned}
$$


The above system of averaged equations is consistent with the system of Euler-Lagrange equations which for such two independent functions have the form

$u_{1}\left(X_{1}, t\right) \Rightarrow \frac{\partial}{\partial t}\left(\frac{\partial L}{\partial \dot{u}_{1}}\right)+\frac{\partial}{\partial X_{1}}\left(\frac{\partial L}{\partial u_{1,1}}\right)-\frac{\partial L}{\partial u_{1}}=0$,
$u_{2}\left(X_{1}, t\right) \Rightarrow \frac{\partial}{\partial t}\left(\frac{\partial L}{\partial \dot{u}_{2}}\right)+\frac{\partial}{\partial X_{1}}\left(\frac{\partial L}{\partial u_{2,1}}\right)-\frac{\partial L}{\partial u_{2}}=0$

where $L=K-W\left(e_{1}, e_{2}, u_{2}\right)$ is the Lagrangian function, $\mathrm{K}$ - kinetic energy and $\mathrm{W}$ - internal elastic energy is function of both strains $e_{1}, e_{2}$ and displacement $\mathrm{u}_{2}$. After some calculations we finally obtain the form. (The prime indicates differentiation with respect to $X_{1}$ ).

$S^{\prime}=\rho_{1} \ddot{u}_{1}, \quad Q^{\prime}-P=\rho_{2} \ddot{u}_{2}$, with $S=\frac{\partial W}{\partial u_{1,1}}, Q=\frac{\partial W}{\partial u_{2,1}}, P=\frac{\partial W}{\partial u_{2}}$

The comparison of the two equivalent equations forms gives the following relationships between the stresses and averaged values and densities

$$
\begin{aligned}
& S=\frac{1}{2 h} \int_{-h}^{h} T_{R 11} d X_{2}, Q=\frac{1}{2 h} \int_{-h}^{h} X_{2} T_{R 21} d X_{2}, P=\frac{1}{2 h} \int_{-h}^{h} T_{R 22} d X_{2}, \\
& \rho_{1}=\rho_{R}, \quad \rho_{2}=\rho_{R} \frac{h^{2}}{3}
\end{aligned}
$$

The averaging of the stresses over the cross section, gives the system of three forces $P, Q, S$ and every such quantity is function of two variables; variable $X_{1}$, and internal scalar variable $h$ - half thickness of the layer.

\section{APPLICATION FOR SHOCK ANALYSIS}

Very important for the study of shock propagation in 1-D are the kinematical conditions of compatibility (Chen (1971), Fu and Scott (1989)) whichever of them can be derived from:

$\frac{d}{d t}[f]=[\dot{f}]+U_{N}\left[f_{X_{1}}\right], \quad \frac{d}{d t}=U_{N} \frac{d}{d X_{1}}$

The space derivative $\mathrm{d} / \mathrm{d} X_{1}$ following the wave front $\Sigma\left(X_{1}, t\right)$ is related to the displacement derivative $\mathrm{d} / \mathrm{dt}$ by $(12)_{2}, U_{N}$ is the normal shock speed. Replacing $f\left(X_{1}, \mathrm{t}\right)$ in $(12)$ in turn by: $u_{1}, \quad v_{p}=\dot{u}_{p}$ and $v_{p, X_{1}}=\ddot{u}_{p}^{\prime}$ we obtain through continual use of $(12)_{1}$ relation which are very useful for transformation of the equation of motion. 
From the equation expressing the balance of momentum on the discontinuity surface and assuming that only two components of the deformation gradient $\left[x_{11}\right] \neq 0$ and $\left[x_{21}\right] \neq 0$ suffer a jump on $\Sigma\left(X_{1}, t\right)$ we obtain (see (5)) the expression for the shock wave speed .

$$
U_{N}^{2}=\frac{\left[T_{R 11}\right]\left[x_{11}\right]+\left[T_{R 21}\right]\left[x_{21}\right]}{\left[x_{11}\right]^{2}+\left[x_{21}\right]^{2}}
$$

The entropy jump $[\eta]([\eta]>0$ for stable shocks) can be calculated from the balance laws on the discontinuity surface (Wesołowski (1978)), because $\left(T_{R i \alpha}\right)^{F}=0$

$$
2 \rho_{R} U_{N}[\sigma]+\left[T_{R i \alpha}\right]\left[\dot{x}_{i}\right] N_{\alpha}=-2\left(T_{R i \alpha}\right)^{F} N_{\alpha}\left[\dot{x}_{i}\right] \Rightarrow[\sigma]=\frac{\left[T_{R i \alpha}\right]}{2 \rho_{R}}\left[x_{i \alpha}\right]
$$

The averaged equations of motion are satisfied on both sides of the discontinuity surface $\Sigma\left(X_{1}, t\right)$.Differentiating these equations with respect to $\mathrm{X}_{1}$ we obtain

$S^{\prime}=\rho_{1} \ddot{u}_{1}, \quad Q^{\prime}-P=\rho_{2} \ddot{u}_{2}$, and $S^{\prime \prime}=\rho_{1} \ddot{u}_{1}^{\prime}, Q^{\prime \prime}-P^{\prime}=\rho_{2} \ddot{u}_{2}^{\prime}$,

On taking the jumps of last two above equations across $\Sigma\left(X_{1}, t\right)$ we finally obtain the basic system of equations for the analysis.

$[S]=\rho_{1}\left[\ddot{u}_{1}\right], \quad\left[Q^{\prime}\right]-[P]=\rho_{2}\left[\ddot{u}_{2}\right]$,

Using the compatibility conditions for the jumps of the right hand sides of them we can obtain system of equations for shock amplitudes and the amplitudes of higher order discontinuities which accompany the shock.

\section{SELECTED PARTICULAR CASES}

Analysis of the problem is restricted to a special kind of second order elastic material, called Murnaghan material

$$
\begin{aligned}
W\left(e_{1}, e_{2}, u_{2}\right)=\rho_{R} \sigma & =\frac{1+2 m}{24}\left(I_{1}-3\right)^{3}+\frac{\lambda+2 \mu+4 m}{8}\left(I_{1}-3\right)^{2}+\frac{8 \mu+n}{8}\left(I_{1}-3\right) \\
& -\frac{m}{4}\left(I_{1}-3\right)\left(I_{2}-3\right)-\frac{4 \mu+n}{8}\left(I_{2}-3\right)+\frac{n}{8}\left(I_{3}-1\right)
\end{aligned}
$$

where $\mathrm{I}_{1}=\mathrm{B}_{i i}, \mathrm{I}_{2}=\left(\mathrm{B}_{i i} \mathrm{~B}_{j j}-\mathrm{B}_{i j} \mathrm{~B}_{i j}\right) / 2, \mathrm{I}_{3}=\operatorname{det}\left(\mathrm{B}_{i j}\right)$ are the invariants of the left Cauchy-Green strain tensor $\mathrm{B}_{i j}, \lambda$ and $\mu$ are Lamé coefficients, and $l, m, n$ are the elastic constants of second order.

On $\Sigma\left(X_{1}, t\right)$ all functions depend on one single variable $X_{1}$, the deformation gradient has the form (5) and we seek perturbation solutions in the form: 
$\begin{array}{lll}{\left[e_{1}\right]=\varepsilon Y_{1}+\varepsilon^{2} Y_{2}+. .,} & {\left[e_{1,1}\right]=Z_{0}+\varepsilon Z_{1}+. .,} & {\left[e_{1,11}\right]=W_{0}+\varepsilon W_{1}+\ldots} \\ {\left[e_{2}\right]=\varepsilon \hat{Y}_{1}+\varepsilon^{2} \hat{Y}_{2}+. .,} & {\left[e_{2,1}\right]=\hat{Z}_{0}+\varepsilon \hat{Z}_{1}+. .,} & {\left[e_{2,11}\right]=\hat{W}_{0}+\varepsilon \hat{W}_{1}+. .}\end{array}$

where $\varepsilon$ is a small dimensionsless perturbation parameter which can be taken to be initial shock strength and we assume the boundary conditions at $\mathrm{X}_{1}=0$

$\begin{array}{ll}{\left[e_{1}\right]_{\hat{X}_{1}=0}=\hat{h} \Rightarrow \varepsilon Y_{1}(0) \approx \hat{h},} & \left.\left[e_{1,1}\right]\right]_{\hat{X}_{1}=0}=k \Rightarrow Z_{0}(0) \approx k, \\ \left.\left[e_{2}\right]\right]_{X_{1}=0}=0 \Rightarrow \varepsilon \hat{Y}_{1}(0) \approx 0, & {\left[e_{2,1}\right]_{X_{1}=0}=0 \Rightarrow \hat{Z}_{0}(0) \approx 0}\end{array}$

Substituting these expansions into the basic system of equations we obtain the uncoupled (with the transverse motion) system of equations for functions connected with the longitudinal motion only. Equating the coefficients of like power $\varepsilon^{0} \varepsilon^{1}$ we have:

$\varepsilon^{1} \Rightarrow \frac{1}{4} c_{1} Y_{1}\left(X_{1}\right) Z_{0}\left(X_{1}\right)+\frac{d Y\left(X_{1}\right)}{d X_{1}}=0, \quad \varepsilon^{0} \Rightarrow \frac{1}{2} c_{1} Z_{0}^{2}\left(X_{1}\right)+\frac{d Z_{0}\left(X_{1}\right)}{d X_{1}}=0$

where $c_{1}=\frac{(3 \lambda+6 \mu+2 l+4 m)}{(\lambda+2 \mu)}$

There are exactly the equations and solutions obtained (Fu and Scott, (1989)) after the application of the simplest theory for nonlinear wave propagation in a straight uniform rod.

$$
\left[e_{1,1}\right]=\frac{k}{1+\frac{c_{1} k X_{0}}{2} X_{1}}, \quad\left[e_{1}\right]=\frac{\hat{h}}{\sqrt{1+\frac{c_{1} k X_{0}}{2} X_{1}}}
$$

For the transverse motion the equations are partly coupled with the longitudinal motion and the first two terms in expansions have the form

$$
\left[e_{2,1}\right] \approx \hat{Z}_{0}=0,\left[e_{2,11}\right] \approx \hat{W}_{0}\left(X_{1}\right)=-\frac{\lambda}{\lambda+\mu} \frac{3}{h^{2}} Z_{0}\left(X_{1}\right)=-\frac{\lambda}{\lambda+\mu} \frac{3}{h^{2}} \frac{k}{1+\frac{c_{1} k X_{0}}{2} X_{1}}
$$

For the isentropic approximation used here the entropy condition is satisfied and the shock is stable. According to our perturbation procedure, from the fact that $e_{2}^{F}=e_{2,1}^{F}=0 \Rightarrow\left[e_{2,1}\right]=e_{2,1}^{B}$ and that the jump $\left[e_{2,1}\right] \approx \hat{Z}_{0}=0$, it follows that on the discontinuity surface (where all the functions depend on one single co-ordinate $X_{1}$ only) the function $\left[e_{2}\right]$ remains unchanged $\left[e_{2}\right] \approx \varepsilon \hat{Y}_{1}(0)=\varepsilon \hat{Y}_{1}\left(X_{1}\right)=$ const, 


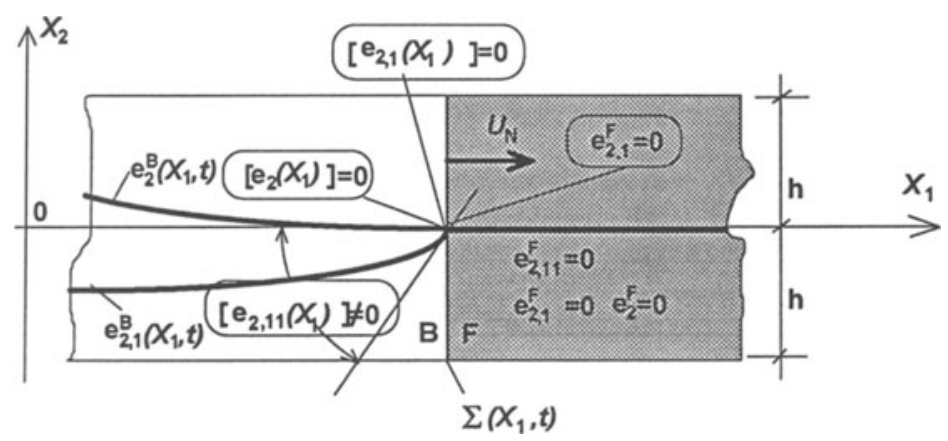

Figure 1 Third order discontinuity $\left[e_{2,11}\right]$ corresponding with the transverse motion which accompany the main longitudinal discontinuity of first order

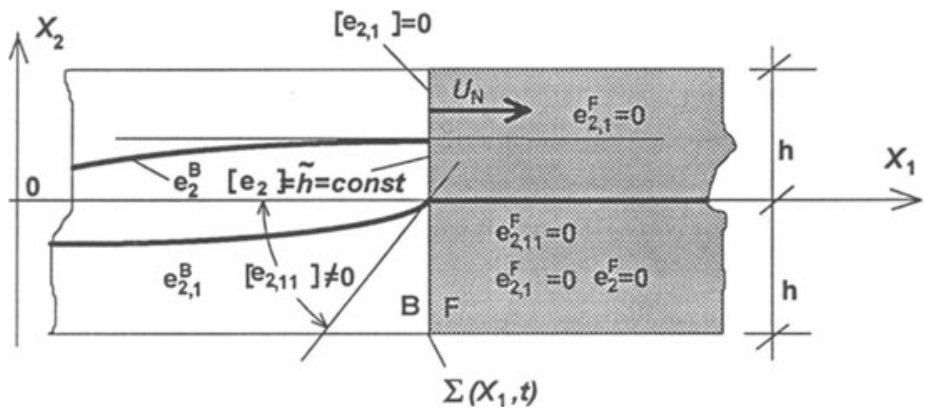

Figure 2 Constant discontinuity of first order $\left[e_{2}\right]=\tilde{h}=$ const and the third order discontinuity $\left[e_{2,11}\right]$ caused by transverse motion coupled with the main longitudinal motion.

Assuming now (see Figure 2) that the initial longitudinal shock $\hat{h}$ is accompanied by the transverse one $\tilde{h}$ i.e.

$$
\left.\left[e_{2}\right]\right|_{X_{1}=0}=\tilde{h} \Rightarrow \varepsilon \hat{Y}_{1}(0) \approx \tilde{h},\left.\quad\left[e_{2,1}\right]\right|_{X_{1}=0}=0 \Rightarrow \hat{Z}_{0}(0) \approx 0
$$

When a shock is initiated by longitudinal loading only the relationship between the initial shock values in the boundary conditions can be given as $T_{R 21}(\hat{h}, \tilde{h})=0$. 


\section{REFERENCES}

Chen, P.J. (1971) One dimensional shock waves in elastic non-conductors. $A R M A, 43,350-$ 362.

Fu, Y.B.and Scott, N.H. (1989) The evolution law of one-dimensional weak nonlinear shock waves in elastic non-conductors. Q.Jl Mech. Appl. Math., 42, 23-39.

Kosiński, S. (1995) Transport equation for shock strength in hyperelastic rods. Engineering Transactions 43, 205-224

Wesołowski, Z. (1978) Strong discontinuity wave in initially strained elastic medium. Archives of Mechanics, 30, 309-322.

Wright, T.W. (1981) Nonlinear waves in rods, in Proceedings of the IUTAM Symposium on Finite Elasticity, Lehigh University,August 10-15,1980 (ed. D.E.Carlsohn and R.T.Shield) 423-443, Martinus Nijhoff Publishers, The Hague/Boston/London 